\title{
Multi-GNSS Induced Performance Enhancements in Constrained Environments
}

\author{
Wenzhe Wang ${ }^{1, *}$, Fengyu $\mathrm{Chu}^{2}$, and Ming Yang $^{3}$ \\ ${ }^{1}$ Department of Geomatics, National Cheng Kung University, Tainan, Taiwan \\ ${ }^{2}$ Department of Geomatics, National Cheng Kung University, Tainan, Taiwan \\ ${ }^{3}$ Department of Geomatics, National Cheng Kung University, Tainan, Taiwan
}

\begin{abstract}
Nowadays, three global navigation satellite systems (GNSS), namely GPS, GLONASS and China's BeiDou System (BDS), are fully-operational in the Asia-Pacific region. Furthermore, the European Galileo system and the Japanese Quasi Zenith Satellite System (QZSS), which is a regional navigation satellite system (RNSS), jointly provide 4 to 8 additional visible satellites in the region. Thus, it is expected that a combination of the above five systems will improve positioning performance as a result of enhanced satellite availability provided by multi-GNSS. In this research, we develop a method to combine GPS, GLONASS, BDS, Galileo, and QZSS pseudorange and carrier phase observations, and investigate positioning performance improvements brought by multi-GNSS. Experimental data were collected in Southern Taiwan to perform pseudorange-based, meter-level absolute (point) positioning as well as carrier phase-based, centimeter-level relative positioning. Test results indicate that (1) using multi-GNSS can effectively improve the accuracy of absolute (single point) and relative positioning, particularly in highly-masked, constrained environments, such as urban areas; (2) combining the five constellations can significantly shorten the TimeTo-First-Fix (TTFF) for rapid ambiguity resolution required by Real-Time Kinematic (RTK) applications in constrained environments.
\end{abstract}

\section{Introduction}

Since its full operation, GPS (global positioning system) has been the most widely used navigation satellite system $([1,2])$. Other satellite positioning systems, e.g., the Russian GLONASS system, the European Galileo system, the Chinese Beidou system (BDS), and the Japanese Quasi Zenith Satellite System (QZSS) are also in rapid development nowadays. It is advantageous to utilize multiple GNSS constellations (Multi-GNSS) to provide more continuous and accurate positioning services for user ends compared with using a single GNSS system alone ([3-7]).

As satellite positioning usually requires an open-sky environment, positioning in constrained environments (such as dense urban districts) has always been difficult when using single GNSS system since only limited number of visible satellites can be used in these areas. Thus it is expected that combining multi-GNSS systems may improve the positioning performance when environments are highly-masked.

In this research, a multi-GNSS positioning model was developed by combining observations from GPS, GLONASS, Galileo, BDS, and QZSS systems. Based on this model, we perform pseudorange-based single point positioning and carrier phase-based, relative positioning in order to analysis the performance improvements induced by using multi-GNSS for positioning in constrained environments.

\section{Multi-GNSS Positioning}

Considering the number and health condition of on-orbit satellites, we use five GNSS constellations for multiGNSS positioning. Measurements (psuedoranges and carrier phases) from GPS, GLONASS, Galileo, BDS, and QZSS are adopted to establish multi-GNSS single point positioning model and relative positioning model. The flow of data processing is shown in Figure 1.

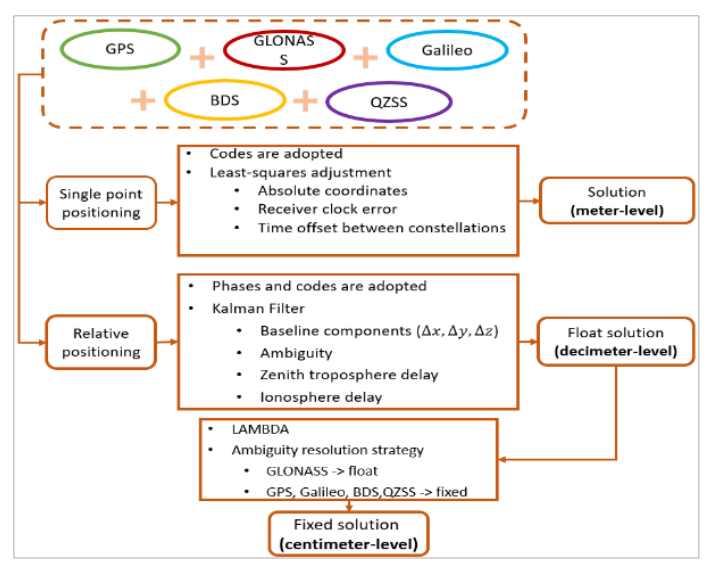

Fig. 1. Date Process Flow

\footnotetext{
*Corresponding author: myang@ mail.ncku.edu.tw
} 


\subsection{Single Point Positioning}

In multi-GNSS single point positioning, pseudorange measurements from GPS, GLONASS, Galileo, BDS, and QZSS are used. At one epoch, the pseudorange measurements can be expressed as:

$$
\left\{\begin{array}{c}
\rho_{G P S}=\sqrt{\left(x_{u}-X^{G}\right)^{2}+\left(y_{u}-Y^{G}\right)^{2}+\left(z_{u}-Z^{G}\right)^{2}} \\
+c \cdot d t_{G P S}+\varepsilon \\
\rho_{G L O}=\sqrt{\left(x_{u}-X^{L}\right)^{2}+\left(y_{u}-Y^{L}\right)^{2}+\left(z_{u}-Z^{L}\right)^{2}} \\
+c \cdot d t_{G P S}+G G T O+\varepsilon \\
\rho_{G A L}=\sqrt{\left(x_{u}-X^{A}\right)^{2}+\left(y_{u}-Y^{A}\right)^{2}+\left(z_{u}-Z^{A}\right)^{2}} \\
+c \cdot d t_{G P S}+G E T O+\varepsilon \\
\rho_{B D S}=\sqrt{\left(x_{u}-X^{B}\right)^{2}+\left(y_{u}-Y^{B}\right)^{2}+\left(z_{u}-Z^{B}\right)^{2}} \\
+c \cdot d t_{G P S}+G B T O+\varepsilon \\
\rho_{Q Z S}=\sqrt{\left(x_{u}-X^{Q}\right)^{2}+\left(y_{u}-Y^{Q}\right)^{2}+\left(z_{u}-Z^{Q}\right)^{2}} \\
+c \cdot d t_{G P S}+G Q T O+\varepsilon
\end{array}\right.
$$

In (1), $\rho_{G P S}, \rho_{G L O}, \rho_{G A L}, \rho_{B D S}, \rho_{Q Z S}$ are the pseudorange measurements (with satellite clock offset, ionospheric and tropospheric delay removed) from GPS, GLONASS, Galileo, BDS, and QZSS system; $\left(x_{u}, y_{u}, z_{u}\right)$ is the user position; $\left(X^{G}, Y^{G}, Z^{G}\right),\left(X^{L}, Y^{L}, Z^{L}\right),\left(X^{A}, Y^{A}, Z^{A}\right)$, $\left(X^{B}, Y^{B}, Z^{B}\right),\left(X^{Q}, Y^{Q}, Z^{Q}\right)$ are positions of GPS, GLONASS, Galileo, BDS, and QZSS satellites; $d t_{G P S}$ is receiver clock error with respect to GPS time; GGTO,GETO,GBTO,GQTO are the between-systembiases of GLONASS, Galileo, BDS and QZSS with respect to GPS; $\varepsilon$ is random noise error in pseudorange observation; $\mathrm{c}$ is the speed of light. By linearizing equation (1), the multi-GNSS single point positioning model can be expressed in a linear form:

$$
\Delta \boldsymbol{\rho}_{n \times 1}=\boldsymbol{H}_{n \times 8} \boldsymbol{x}_{8 \times 1}+\boldsymbol{\varepsilon}_{8 \times 1}
$$

In (2), $\Delta \boldsymbol{\rho}_{n \times 1}=\boldsymbol{\rho}_{n \times 1}-\boldsymbol{\rho}_{n \times 1}^{0}, \boldsymbol{\rho}_{n \times 1}$ are the $n$ pseudorange measurements at the epoch, $\boldsymbol{\rho}_{n \times 1}^{0}$ are the corresponding computed values of pseodoranges, $\boldsymbol{\rho}_{i \times 1}^{0}=$ $\sqrt{\left(x_{\text {user }}^{0}-X^{i}\right)^{2}+\left(y_{\text {user }}^{0}-Y^{i}\right)^{2}+\left(z_{\text {user }}^{0}-Z^{i}\right)^{2}}(1 \leq i \leq n$, while $i$ is an integer), where $\left(x_{\text {user }}^{0}, y_{\text {user }}^{0}, z_{\text {user }}^{0}\right)$ is the approximate user position and $\left(X^{i}, Y^{i}, Z^{i}\right)$ is the position of the $i$-th satellite, $\boldsymbol{H}_{n \times 8}$ is the coefficient matrix that can be written as:

$\boldsymbol{H}_{n \times 8}=\left|\begin{array}{cccccc}\boldsymbol{G}_{n_{G P S} \times 3} & \boldsymbol{I}_{n_{G P S} \times 1} & 0 & 0 & 0 & 0 \\ \boldsymbol{G}_{n_{G L O} \times 3} & \boldsymbol{I}_{n_{G L O} \times 1} & \boldsymbol{I}_{n_{G L O} \times 1} & 0 & 0 & 0 \\ \boldsymbol{G}_{n_{G A L} \times 3} & \boldsymbol{I}_{n_{G A L} \times 1} & 0 & \boldsymbol{I}_{n_{G A L} \times 1} & 0 & 0 \\ \boldsymbol{G}_{n_{B D S} \times 3} & \boldsymbol{I}_{n_{B D S} \times 1} & 0 & 0 & \boldsymbol{I}_{n_{B D S} \times 1} & 0 \\ \boldsymbol{G}_{n_{Q Z S} \times 3} & \boldsymbol{I}_{n_{Q Z S} \times 1} & 0 & 0 & 0 & \boldsymbol{I}_{n_{Q Z S} \times 1}\end{array}\right|$

$\boldsymbol{G}$ is the geometry matrix between satellite and receiver, $\boldsymbol{G}_{\boldsymbol{i} \times \mathbf{3}}=\left|\begin{array}{lll}\frac{x_{\text {user }}^{0}-X^{i}}{\boldsymbol{\rho}_{i}^{0}} & \frac{y_{u s e r}^{0}-Y^{i}}{\boldsymbol{\rho}_{i}^{0}} & \frac{z_{\text {user }}^{0}-Z^{i}}{\boldsymbol{\rho}_{i}^{0}}\end{array}\right|(1 \leq i \leq n$, while $i$ is an integer), $n_{G P S}, n_{G L O}, n_{G A L}, n_{B D S}, n_{Q Z S}$ are the number of pseudorange measurements from GPS, GLONASS, Galileo, BDS and QZSS system $\boldsymbol{x}_{8 \times 1}=$ \begin{tabular}{|llllllll}
$\Delta x_{u}$ & $\Delta y_{u}$ & $\Delta z_{u}$ & $c \cdot d t_{G P S}$ & $G G T O$ & $G E T O$ & $G B T O$ & $\left.G Q T O\right|^{T}$.
\end{tabular} Applying least-squares estimation to (2) yields:

$$
\widehat{\boldsymbol{x}}_{8 \times 1}=\left(\boldsymbol{H}^{\boldsymbol{T}}{ }_{n \times 8} \boldsymbol{H}_{n \times 8}\right)^{-1} \boldsymbol{H}^{\boldsymbol{T}}{ }_{n \times 8} \Delta \boldsymbol{\rho}_{n \times 1}
$$

In multi-GNSS single point positioning, the parameters to be estimated include user position $\left(x_{u}, y_{u}, z_{u}\right)$, receiver clock error with respect to GPS time $\left(d t_{G P S}\right)$, betweensystem-biases (GGTO,GETO,GBTO,GQTO).

\subsection{Relative Positioning}

In multi-GNSS relative positioning, pseudorange measurements from GPS, GLONASS, Galileo, BDS and QZSS, along with carrier phase measurements from GPS, Galileo, BDS and QZSS are adopted. Because the FDMA (Frequency Division Multiple Access) technique used to transmit GLONASS signals may bring difficulties to ambiguity resolution $([8,9])$, carrier phase measurements from GLONASS system are excluded here when establishing relative positioning model for multi-GNSS. At one epoch, the double-differenced pseudorange measurements and carrier phase measurements can be expressed as:

$$
\left\{\begin{array}{c}
\Delta \nabla P^{S}=\Delta \nabla \rho^{S}+\Delta \nabla T^{S}+\Delta \nabla I^{S}+\Delta \nabla \varepsilon_{P}^{S} \\
\Delta \nabla \Phi^{S}=\Delta \nabla \rho^{S}+\lambda^{S} \Delta \nabla N^{S}+\Delta \nabla T^{S}-\Delta \nabla I^{S}+\Delta \nabla \varepsilon_{\Phi}^{S}
\end{array}\right.
$$

In (4), $S$ indicates system type (GPS, GLONASS, Galileo, BDS, QZSS), $P$ and $\Phi$ are pseudorange and carrier phase (in meters) measurements, $\rho$ is the geometric distance between user and satellite, $N$ is the number of integer cycles of carrier phase measurement, $T$ and $I$ are tropospheric and ionospheric delays, respectively, $\varepsilon_{P}$ and $\varepsilon_{\Phi}$ are noise errors in pseudorange and carrier phase measurements. A simplified linearized multi-GNSS positioning model in relative positioning can be given as:

$$
y=A a+B b+\varepsilon
$$

In (4), $\boldsymbol{a}$ denotes the parameter vector of doubledifferenced integer ambiguity, $\boldsymbol{b}$ denotes the parameter vector including baseline components $(\Delta x, \Delta y, \Delta z)$, ionospheric delay and zenith tropospheric delay (ZTD), $\boldsymbol{A}$ and $\boldsymbol{B}$ are the coefficient matrices corresponding to vector $\boldsymbol{a}$ and $\boldsymbol{b}, \boldsymbol{y}$ is the observation vector that includes double-differenced pseudorange observations and carrier phase observations. $\boldsymbol{\varepsilon}$ is the vector that denotes random noise error.

According to (4), in relative positioning, pseudorange and carrier phase measurements from multi-GNSS systems are adopted. By using Kalman filtering, unknown parameters including baseline components $(\Delta x, \Delta y, \Delta z)$, float-value carrier phase ambiguities, zenith tropospheric delay (ZTD) and ionospheric delay can be estimated at each epoch. It should be mentioned that the float-value carrier phase ambiguities need be correctly fixed, so LAMBDA method ([10]) is applied to deccorelate the carrier phase ambiguities. Once correct integer ambiguities of carrier phase measurements from GPS, Galileo, Beidou and QZSS systems are found, centimeterlevel accuracy can be achieved in relation positioning. 


\section{Experiments and Results}

Currently in Taiwan, dual-constellation (GPS/GLONASS) combination are commonly used in positioning ([11]). To investigate the improvements (especially in constrained environments) in positioning performance induced by combining multi-GNSS constellations, experimental data were collected to perform pseudorange-based, meter-level absolute (point) positioning as well as carrier phase-based, centimetrelevel relative positioning. The two experiments were performed in southern Taiwan, on November $15^{\text {th }}, 2017$. Static data were collected for one hour at two stations SPSI and HHLA equipped with Zephyr geodetic antennas and Trimble NetR9 receivers. Precise positions of SPSI and HHLA stations were obtained using GIPSY software in a Precise Point Positioning mode over one-month observation data. Dual-frequency signals collected in this research include GPS L1 and L2C, GLONASS L1 and L2, Galileo E1 and E5, BDS B1 and B2, QZSS L1 and L2. Figure 2 and Figure 3 show satellite the constellations and the number of visible satellites during the test period.

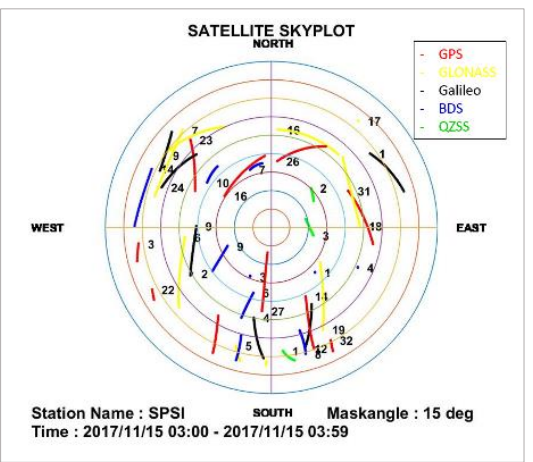

Figure 2. Satellite constellation (Nov $15^{\text {th }}, 2017$ )

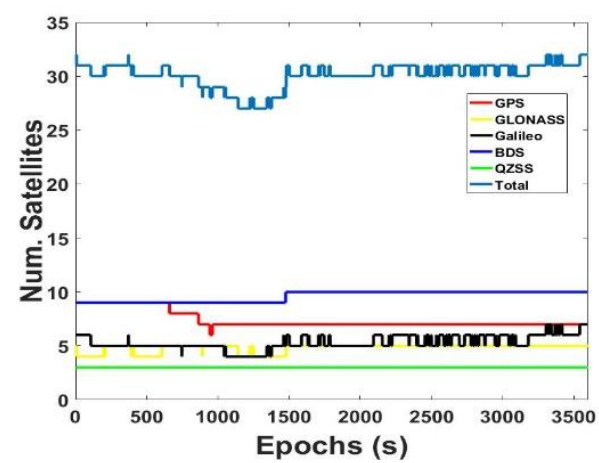

Figure 3. Number of available satellites (cut-off $=15^{\circ}$ )

\subsection{Single Point Positioning Results}

Single point positioning is often used in navigation. In this experiment, one-hour static data was collected in station SPSI to perform multi-GNSS single point positioning. The sampling rate was $1 \mathrm{~Hz}$.

Table 1. Horizontal(H) and vertical(V) mean error/RMS value, single point positioning (in meters)

\begin{tabular}{|c|c|c|c|c|}
\hline & \multicolumn{2}{|c|}{$15^{\circ}$} & \multicolumn{2}{c|}{$35^{\circ}$} \\
\cline { 2 - 5 } & $\mathrm{H}$ & $\mathrm{V}$ & $\mathrm{H}$ & $\mathrm{V}$ \\
\hline GPS/GLONASS & $0.71 / 1.38$ & $0.89 / 1.98$ & $1.23 / 7.50$ & $4.67 / 42.08$ \\
\hline Multi-GNSS & $0.53 / 0.98$ & $0.59 / 1.10$ & $0.78 / 1.93$ & $1.42 / 2.36$ \\
\hline
\end{tabular}

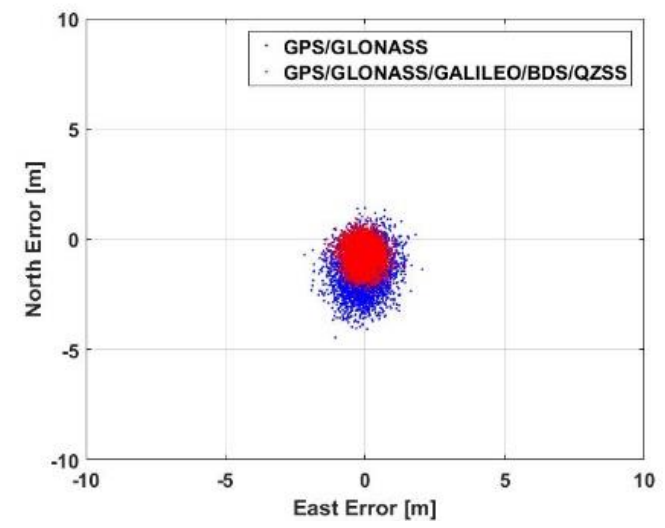

(a) Cut-off $=15^{\circ}$, Horizontal

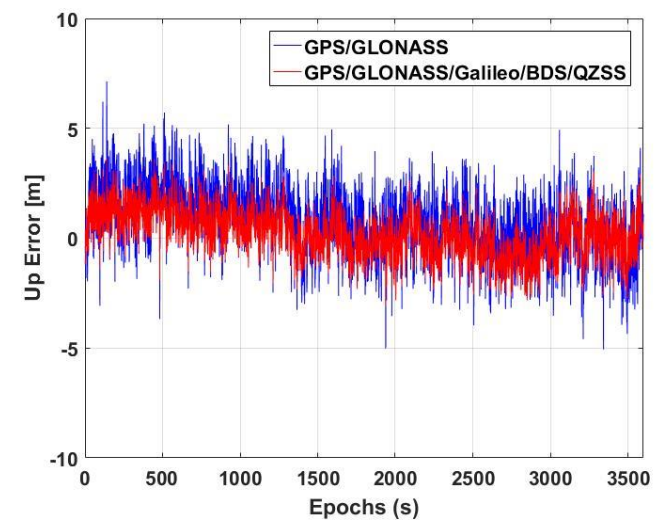

(b) Cut-off $=15^{\circ}$, Vertical

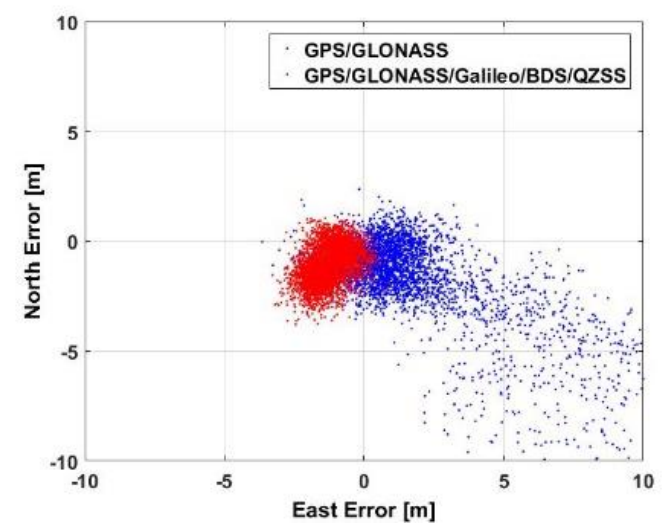

(d) Cut-off $=35^{\circ}$, Horizontal

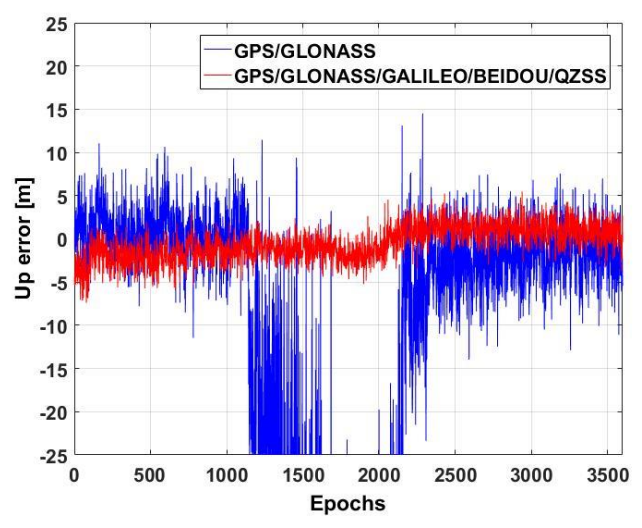

(d) Cut-off $=35^{\circ}$, Vertical

Fig. 4. Single Point Positioning Errors. 
To investigate and compare the positioning performance in normal and constrained environments, positioning results under $15^{\circ}$ and $35^{\circ}$ cut-off angles are analysed in this section. In Table 1, horizontal $(\mathrm{H})$ and vertical (V) mean errors and root mean square (RMS) errors of single point positioning are given for $15^{\circ}$ and $35^{\circ}$ cut-off angle. The result indicates that, mean error and RMS values (in horizontal and vertical directions) are both decreased by using multi-GNSS constellations in single point positioning. It should be mentioned that under $35^{\circ}$ cut-off angle, the positioning accuracy for dual-constellation combination is only $7.50 \mathrm{~m}$ and $42.08 \mathrm{~m}$ in horizontal and vertical directions, while corresponding results of the multi-GNSS combination are $1.93 \mathrm{~m}$ and $2.36 \mathrm{~m}$. The positioning results under $35^{\circ}$ cut-off angle indicate that, using multi-GNSS combination can greatly improve the positioning performance (particularly in the vertical direction) in constrained environments.

In support of better understanding the results of Table 1, the results of single point positioning error are graphically present in Figure 4. This figure consists of $2 \times 2$ subfigures, Figure 4(a) and Figure 4(b) indicate the positioning results under $15^{\circ}$ cut-off angles, Figure 4(c) and Figure 4(d) indicate the positioning results under $35^{\circ}$ cut-off angles. The blue dots and line indicate the horizontal and vertical errors using GPS/GLONASS combination while the red dots and line indicate the horizontal and vertical errors using multi-GNSS combination.

\subsection{Relative Positioning Results}

Relative positioning is often applied in researches on geodesy or earth science and so on. In this experiment, one-hour static data was collected in two stations SPSI and HHLA to perform multi-GNSS relative positioning. The distance between two stations is about $11 \mathrm{Km}$ and the sampling rate was $1 \mathrm{~Hz}$. To investigate and compare the positioning performance in normal and constrained environments, positioning results under $15^{\circ}$ and $35^{\circ}$ cutoff angles are analysed in this section.

Table 2. Horizontal(H) and vertical(V) mean error/RMS value,

\begin{tabular}{|c|c|c|c|c|}
\multicolumn{5}{c}{ relative positioning (in meters) } \\
\cline { 2 - 5 } & $\mathrm{H}$ & $\mathrm{V}$ & $\mathrm{H}$ & $\mathrm{V}$ \\
\hline & $\mathrm{H}$ & $\mathrm{V}$ & \multicolumn{2}{c|}{$35^{\circ}$} \\
\hline GPS/GLONASS & $0.014 / 0.013$ & $0.009 / 0.024$ & $0.011 / 0.018$ & $0.017 / 0.131$ \\
\hline Multi-GNSS & $0.013 / 0.010$ & $0.004 / 0.017$ & $0.008 / 0.011$ & $0.016 / 0.026$ \\
\hline
\end{tabular}

In Table 2, horizontal $(\mathrm{H})$ and vertical $(\mathrm{V})$ mean errors and root mean square (RMS) errors of $11 \mathrm{Km}$ relative positioning are given for $15^{\circ}$ and $35^{\circ}$ cut-off angles. Similar to the results of single point positioning, in relative positioning, mean errors and RMS values (horizontal and vertical directions) are decreased by using multi-GNSS constellations. When under $35^{\circ}$ cut-off angle, the horizontal and vertical RMS values of GPS/GLONASS combination are $0.018 \mathrm{~m}$ and $0.131 \mathrm{~m}$, respectively, while corresponding values of multi-GNSS combination are $0.011 \mathrm{~m}$ and $0.026 \mathrm{~m}$. The results indicate that in relative positioning, centimetre-level accuracy can still be achievable by using multi-GNSS even in high cutoff angle.
The results of relative positioning error are graphically (a) Cut-off $=15^{\circ}$, Horizontal

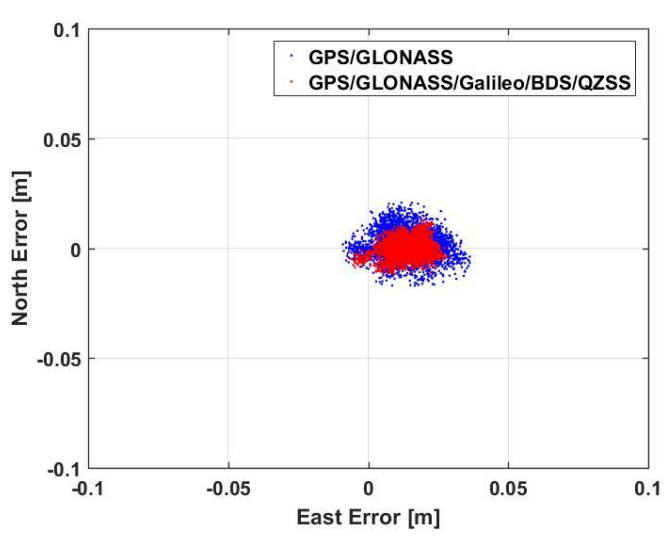

(b) Cut-off $=15^{\circ}$, Vertical

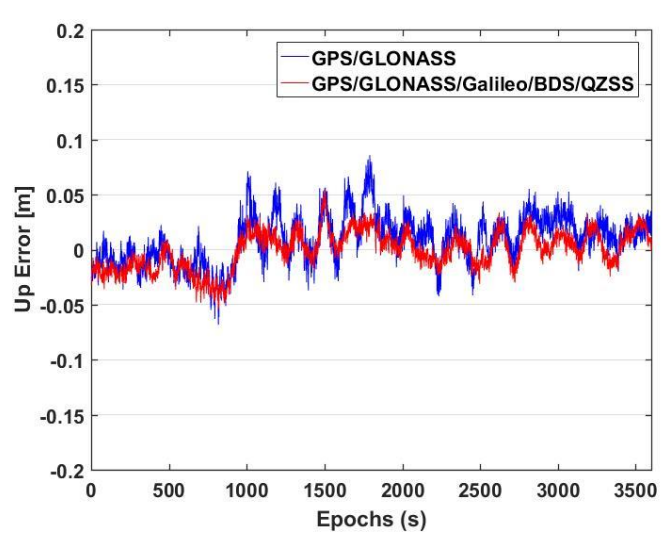

(c) Cut-off $=35^{\circ}$, Horizontal

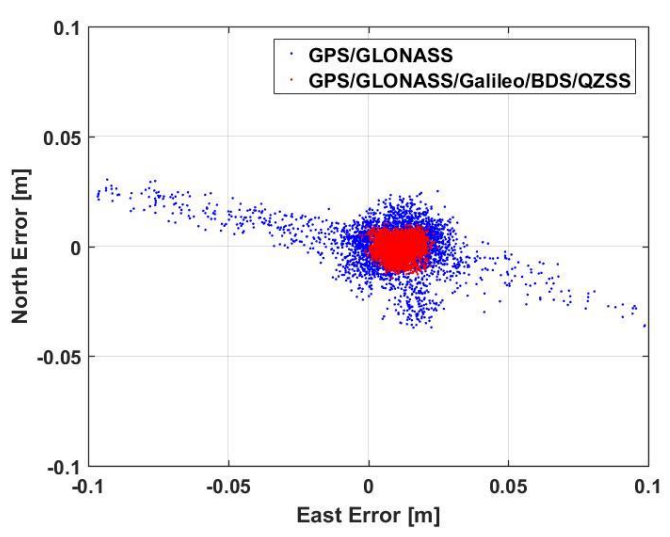

(d) Cut-off $=35^{\circ}$, Vertical

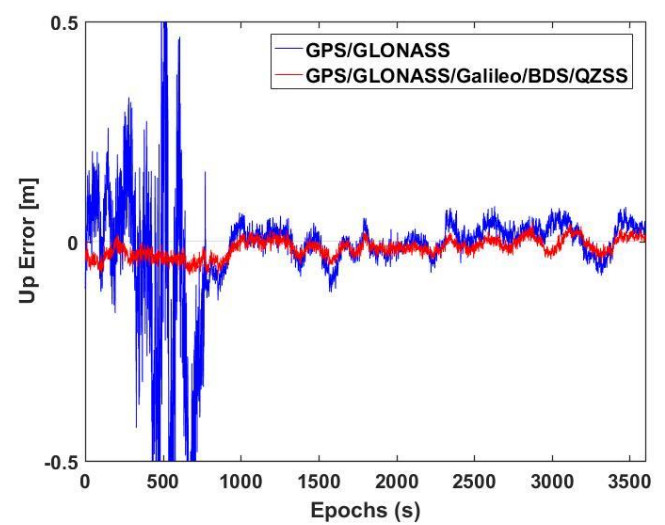


Fig. 5. Relative Positioning Errors.

present in Figure 5. Figure 5 consists of $2 \times 2$ subfigures,

Figure 5(a) and Figure 5(b) indicate the positioning results under $15^{\circ}$ cut-off angles, Figure 5(c) and Figure 5(d) indicate the positioning results under $35^{\circ}$ cut-off angles. The blue dots and line indicate the horizontal and vertical errors using GPS/GLONASS combination while the red dots and line indicate the horizontal and vertical errors using multi-GNSS combination.

In relative positioning, Time-To-First-Fix (TTFF) is an important issue as TTFF value indicates the time for rapid ambiguity resolution required by high-accuracy relative positioning applications, such as Real-Time Kinematic (RTK) in constrained environments. Figure 6 shows the TTFF values in relative positioning, the TTFF values for using GPS/GLONASS combination under $15^{\circ}$ and $35^{\circ}$ cut-off angle are 15 seconds and 25 seconds, while for multi-GNSS, the TTFF values are 4 seconds and 5 seconds, respectively. The result shows that with multiGNSS system, when using multi-GNSS, less time are required to perform centimetre-level positioning. This may benefit some filed works that are in urban area where sky-view is limited.

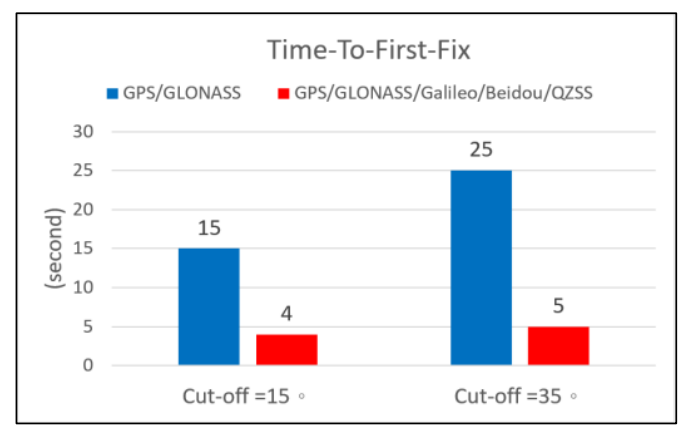

Fig. 6. Time-To-First-Fixing Values.

\section{Conclusions}

In this research, we have adopted a method to fully combine GPS, GLONASS, BDS, Galileo, and QZSS pseudorange and carrier phase observations, and perform single point positioning as well as relative positioning to further investigate positioning performance improvements brought by multi-GNSS. The conclusions are: (1) using multi-GNSS can effectively improve the accuracy of single point and relative positioning, particularly in highly-masked, constrained environments, such as urban areas; (2) combining the five constellations can significantly shorten the Time-To-First-Fix (TTFF) for rapid ambiguity resolution required by Real-Time Kinematic (RTK) applications in constrained environments.

\section{References}

1. E. Kaplan, C J. Hegarty, Understanding GPS: Principles and Applications $\left(2^{\text {nd }}\right.$ Edition. Boston : Artech House, 2006)
2. G. Xie, Principle of GPS and Receiver Design ( Beijing : Publushing House of Electronics Industry, 2009)

3. A. Angrisano, S. Gaglione, C. Gioia, Performance Assessment of GPS/GLONASS Single Point Positioning in an Urban Environment[J]. Acta. Geod. Geophys. 48, 148-161 (2013)

4. C. Weng, C. Chen, W. Ting, Statiostical Characterization of BEIDOU Navigation Errors with a Consumer Multi-constellation GNSS receiver[C]. ION GNSS, Tennessee, (2013)

5. A. Angrisano, S. Gaglione, C. Gioia, Performance A ssessment of Aided Global Navigation Satellite System for Land Navigation[J]. IET Radar, Sonar and Navigation. 671-680(2012)

6. R. Winit, K. O'Keefe, Four-constellation Reliability in challenging GNSS Signal Environments and the Estimation of Inter-system Time-offsets[C]. ION GNSS, Nashivelle, TN, (2013)

7. C. Cai. Y. Gao, A combined GPS/GLONASS Navigation Algorithm for Use with Limited Satellite Visiability[J]. Journal of Navigation. E 62, 671-685 (2009)

8. R.B. Ong, M.G. Petovello, G. Lachapelle, Assessment of GPS/GLONASS RTK under Various Operational Conditions[C]. ION GNSS, Savannah, GA, (2009)

9. L. Wanninger, S. Wallstab-Freitag, Combined Processing of GPS,GLONASS, and SBAS Code Phase and Carrier Phase Measurements[C]. ION GNSS, Fort Worth, TX, (2007)

10. P. J. G. Tuenissen, The Least-squares Ambiguity Deccorrelation Adjustment : A Method for Fast GPS Inter Ambiguity Estimation[J]. Journal of Geodesy. E 72, 606-612 (1998)

11. Tsai, M. L., K. W. Chiang, M. Yang, and H. C. Chen, The accuracy and reliability analysis for future GNSS in Taiwan region, Journal of Photogrammetry and Remote Sensing, 13, 57-64 (2008) 